\title{
Characterization of host response, resorption, and strength properties, and performance in the presence of bacteria for fully absorbable biomaterials for soft tissue repair
}

\author{
N. F. N. Stoikes ${ }^{1}$ J. R. Scott ${ }^{2,3} \cdot$ A. Badhwar ${ }^{3}$ C. R. Deeken ${ }^{4}$ - G. R. Voeller $^{1}$
}

Received: 7 November 2016 / Accepted: 6 August 2017 / Published online: 16 August 2017

(C) The Author(s) 2017. This article is an open access publication

\begin{abstract}
Purpose The objective was to evaluate the host response, resorption, and strength properties, and to assess the performance in the presence of bacteria for Phasix ${ }^{\mathrm{TM}}$ Mesh $\left(\right.$ Phasix $^{\mathrm{TM}}$ ) and Gore ${ }^{\circledR}$ Bio-A ${ }^{\circledR}$ Tissue Reinforcement (Bio$\left.\mathrm{A}^{\circledR}\right)$ in preclinical models.

Methods In a rat model, one mesh $(2 \times 2 \mathrm{~cm})$ was implanted subcutaneously in $n=60$ rats. Animals were euthanized after $2,4,8,12,16$, or 24 weeks $(n=5 /$ mesh/time point), and implant sites were assessed for host inflammatory response and overall fibrotic repair thickness. In a rabbit model, meshes ( $3.8 \mathrm{~cm}$ diameter) were bilaterally implanted in subcutaneous pockets in $n=20$ rabbits ( $n=10$ rabbits/ mesh) and inoculated with $10^{8} \mathrm{CFU}$ clinically isolated methicillin-resistant Staphylococcus aureus (MRSA). One mesh type was implanted per animal. Animals were euthanized after 7 days, and implants were assessed for abscess formation, bacterial colonization, and mechanical strength. Results In the rat study, Phasix ${ }^{\mathrm{TM}}$ and Bio- $\mathrm{A}^{\circledR}$ exhibited similar biocompatibility, although Bio- ${ }^{\circledR}$ demonstrated a significantly greater inflammatory response at 4 weeks compared to Phasix ${ }^{\mathrm{TM}}(p<0.01)$. Morphometric analysis demonstrated rapid resorption of Bio-A ${ }^{\circledR}$ implants with initially
\end{abstract}

N. F. N. Stoikes

nstoikes@uthsc.edu

1 Department of Surgery, University of Tennessee Health Science Center, 6029 Walnut Grove Road, Suite 106, Memphis, TN 38138, USA

2 Department of Molecular Pharmacology, Physiology and Biotechnology, Brown University, Providence, RI 02906, USA

3 C. R. Bard, Inc. (Davol), Warwick, RI 02886, USA

4 Covalent Bio, LLC, St. Louis, MO 63025, USA thicker repair sites at 2, 4, 8, and 12 weeks $(p<0.0001)$, which transitioned to significantly thinner sites compared to Phasix ${ }^{\mathrm{TM}}$ at 16 and 24 weeks $(p<0.0001)$. In the rabbit bacterial inoculation study, Phasix ${ }^{\mathrm{TM}}$ exhibited significantly lower abscess score $(p<0.001)$ and bacterial colonization $(p<0.01)$, with significantly greater mechanical strength than Bio- $\mathrm{A}^{\circledR}(p<0.001)$.

Conclusions Host response, resorption, repair thickness, strength, and bacterial colonization suggest a more stable and favorable outcome for monofilament, macroporous devices such as Phasix ${ }^{\mathrm{TM}}$ relative to multifilament, microporous devices such as Bio- $\mathrm{A}^{\circledR}$ over time.

Keywords Phasix ${ }^{\mathrm{TM}}$ Mesh $\cdot$ Hernia $\cdot$ Infection $\cdot$ MRSA . Gore ${ }^{\circledR}$ Bio-A ${ }^{\circledR}$ Tissue Reinforcement

\section{Introduction}

Biomaterials for soft tissue reconstruction applications such as hernia repair have evolved over the past several decades from the original permanent meshes to include partially resorbable, biologic, and absorbable (synthetic or biologically sourced) meshes. Absorbable synthetic meshes present some important advantages, particularly compared to biologic meshes, including more consistent material characteristics and predictable resorption profiles. Additionally, absorbable synthetic meshes provide more rapid tissue integration than biologic meshes, which may promote more rapid host bacterial clearance if inadvertently contaminated [1].

These issues have led to the evolution of absorbable synthetic meshes which represents a relatively new genre of mesh. Absorbable synthetic meshes can be further subdivided into synthetic versus biologically sourced categories. Synthetic, fully resorbable scaffolds are comprised of materials such as 
polyglycolic acid (Dexon ${ }^{\mathrm{TM}}$ Mesh, Covidien, Plc., Mansfield, MA), a copolymer of glycolide and lactide (Vicryl ${ }^{\mathrm{TM}}$ Mesh, Ethicon, Inc., Somerville, NJ), a copolymer of polyglycolide/ polylactide/polytrimethylene carbonate-polylactide/polytrimethylene carbonate (TIGR ${ }^{\circledR}$ Matrix, Novus Scientific, Uppsala, Sweden), and a copolymer of poly (glycolide:trimethylene carbonate) (Gore ${ }^{\circledR}$ Bio-A ${ }^{\circledR}$ Tissue Reinforcement, W.L. Gore $\&$ Associates, Inc., Flagstaff, AZ). Biologically sourced, fully resorbable scaffolds are comprised of materials derived from silkworms (SERI ${ }^{\circledR}$ Surgical Scaffold, Allergan, Inc., Irvine, CA) or poly-4-hydroxybutyrate derived from genetically modified K12 E. coli bacteria (Phasix ${ }^{\mathrm{TM}}$ Mesh, C.R. Bard, Inc./ Davol, Inc., Warwick, RI).

The overall resorption profile of the scaffold is driven primarily by the material composition of the device. Some fully resorbable components are resorbed over a relatively short period (i.e., Vicryl ${ }^{\mathrm{TM}}$ Mesh and Bio-A ${ }^{\circledR}$, approximately 6 months), while others possess a longer term resorption profile (i.e., TIGR $^{\circledR}, \mathrm{SERI}^{\circledR}$, Phasix $^{\mathrm{TM}}$ ) that may be more desirable in hernia repair applications in which the native tissue must be given adequate time to heal and integrate with the scaffold. Morphological differences such as the number of filaments (i.e., monofilament versus multifilament) and pore structure (i.e., macroporous versus microporous) may also impact the resorption profile and host tissue response due to the resulting surface area of the scaffold [2-6]. As such, a microporous, multifilament structure presents the host with a foreign body of greater surface area than a macroporous, monofilament design. The existing literature suggests that scaffolds with greater surface area may be associated with a more pronounced inflammatory response and reduced resistance to bacterial colonization [2-6], but comparisons of absorbable synthetics are still lacking given the sparsity and recent release of available products.

The objective of this study was to evaluate the host tissue response, resorption profile, repair thickness, strength, and the performance in the presence of bacteria associated with a macroporous, monofilament, fully resorbable scaffold design (Phasix ${ }^{\mathrm{TM}}$ ) compared to a microporous, multifilament, fully resorbable scaffold design (Bio- $\mathrm{A}^{\circledR}$ ) in a medium-term, non-contaminated hernia defect rat model to evaluate host response, mesh resorption, and repair thickness and a shortterm, rabbit bacterial inoculation model to evaluate mesh strength and performance in the presence of bacteria.

\section{Methods}

\section{Non-contaminated hernia defect model (rat study)}

\section{Study design and materials}

A non-contaminated hernia defect model with subcutaneous mesh implantation was approved by the Institutional
Animal Care and Use Committee at CBSET, Inc. (Lexington, MA). All animals were treated in accordance with the Guide for the Care and Use of Laboratory Animals. The study consisted of $60(n=60)$ male Sprague-Dawley rats (332.7-483.6 g, 8 weeks old), divided into 2 groups, including fully resorbable synthetic [Gore ${ }^{\circledR}$ Bio-A ${ }^{\circledR}$ Tissue Reinforcement (Bio- $\left.\left.{ }^{\circledR} ; n=30\right)\right]$ and fully resorbable biologically derived [Phasix ${ }^{\mathrm{TM}}$ Mesh (Phasix ${ }^{\mathrm{TM}} ; n=30$ )]. The structural differences between these biomaterials are depicted in scanning electron micrographs in Fig. 1. Phasix ${ }^{\mathrm{TM}}$ is comprised of a monofilament, macroporous design of poly-4-hydroxybutyrate derived from genetically modified K12 E. coli bacteria, whereas Bio- ${ }^{{ }^{\circledR}}$ is comprised of a multifilament, microporous, sheet-like design of poly(glycolide:trimethylene carbonate).

\section{Device implantation/explantation}

Meloxicam ( $1 \mathrm{mg} / \mathrm{kg}$, PO or SQ) was administered on the morning of implantation, and isoflurane gas inhalant anesthesia was delivered at $0.25-5 \%$ in oxygen, to effect. Buprenorphine $(0.02 \mathrm{mg} / \mathrm{kg}$, SQ) was also administered at the time of anesthetic induction. The animal was then placed in dorsal recumbency, and the surgical site was shaved, prepared, and draped aseptically. Using sterile technique, a 3-5-cm midline incision was made through the skin of the ventral abdomen (Fig. 2a). The skin on the right side of the abdomen was bluntly dissected to reveal the subcutaneous plane. A $0.5-\mathrm{cm}$ defect was created in the muscle layer using a surgical scalpel without penetrating the peritoneal cavity (Fig. 2b, c). A $2 \times 2 \mathrm{~cm}$ square piece of mesh was soaked/ rinsed according to the instructions for use (as appropriate) and then implanted into the subcutaneous plane and fixed over the surgical defect using four interrupted 5-0 Prolene ${ }^{\mathrm{TM}}$ sutures, one at each corner (Fig. 2d). The skin was closed with continuous 4-0 Vicryl ${ }^{\mathrm{TM}}$ sutures in a subcuticular fashion, followed by skin staples or wound clips. Animals were monitored during recovery until upright and ambulatory. Meloxicam (1 mg/kg, PO) was administered every $24 \mathrm{~h}$ for the first three postoperative days. Co-Flex ${ }^{\circledR}$ or ELASTIKON ${ }^{\circledR}$ wrapping material was used to prevent disruption of the surgical incision site. Animals were euthanized at $2,4,8,12,16$, or 24 weeks ( $n=5$ animals of each mesh type at each time point) via carbon dioxide asphyxiation in accordance with the American Veterinary Medical Association (AVMA) guidelines.

\section{Inflammation}

Following euthanasia, the skin over the implant site was reflected, and the implant site was photographed, excised, and immersion-fixed in $10 \%$ neutral, buffered formalin. 

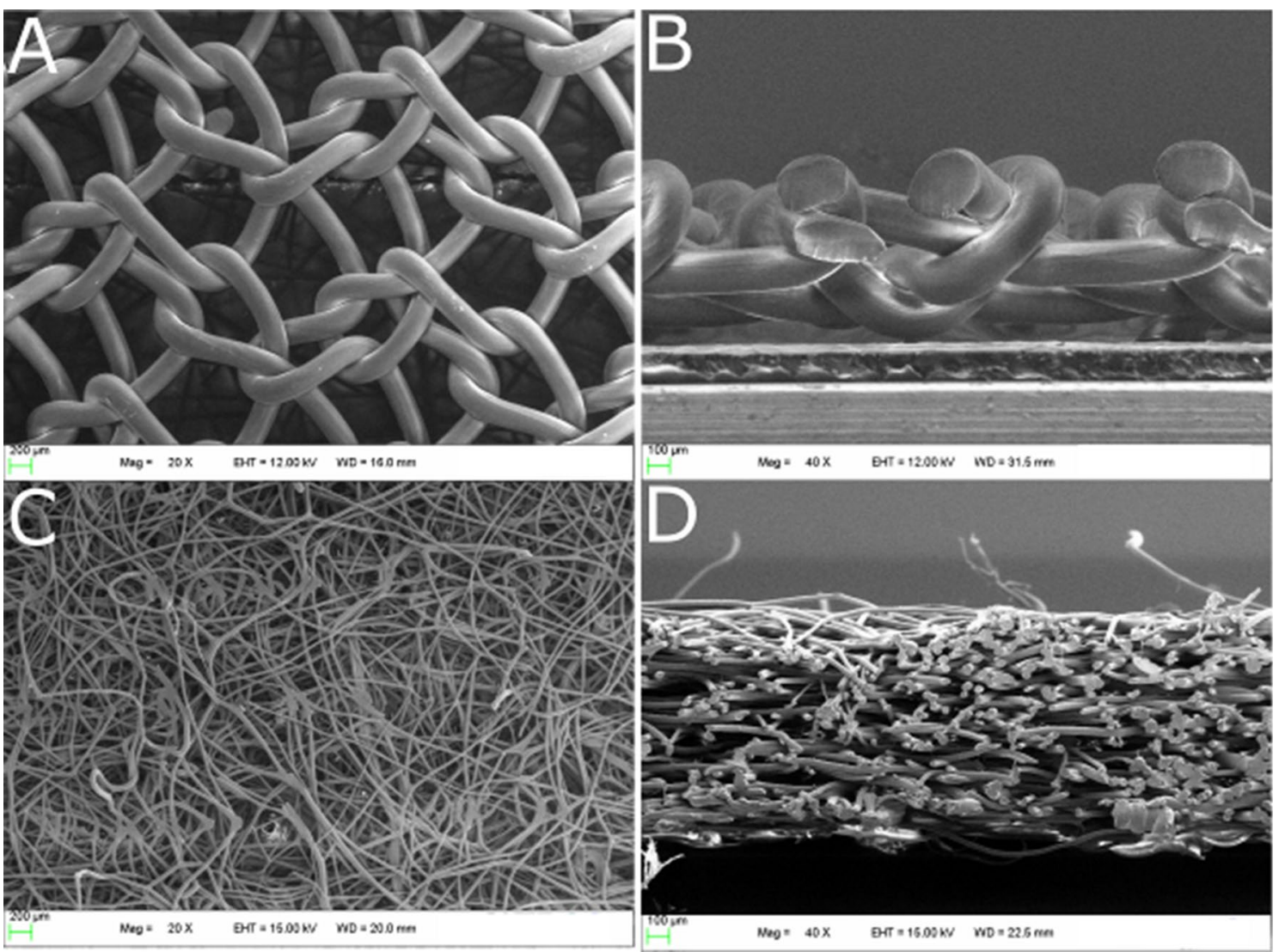

Fig. 1 Scanning electron micrographs: a top view of Phasix ${ }^{\mathrm{TM}}$ at $\times 20$ magnification (scale bar $200 \mu \mathrm{m})$, b side view of Phasix ${ }^{\mathrm{TM}}$ at $\times 40$ magnification (scale bar $100 \mu \mathrm{m}$ ), $\mathbf{c}$ top view of Bio- $\mathrm{A}^{\circledR}$ at $\times 20$ mag-

Specimens were then processed, embedded in paraffin, sectioned at $5 \mu \mathrm{m}$, and stained with Masson's trichrome and hematoxylin and eosin. Inflammatory response was scored by a blinded, board-certified veterinary pathologist as described previously $[7,8]$ : $0=$ no response, $1=$ minimal $/$ barely detectable, $2=$ mild/slightly detectable, $3=$ moderate/easily detectable, and $4=$ marked/very evident.

\section{Repair thickness}

Morphometric analysis was also conducted on the Masson's Trichrome stained slides for one representative section per implant. At low magnification, a 6-mm segment of the implant was selected and evaluated for repair thickness, which was defined as the sum of the mesh thickness and the surrounding collagenous material, measured in millimeters (mm). Repair thickness measurements were taken at approximately $0.5 \mathrm{~mm}$ increments along the length of the cross section, resulting in a total of $13(n=13)$ measurements per section. The contribution of the thickness of the mesh itself to these measurements was also assessed on three $(n=3)$ measurements per section. Repair thickness nification (scale bar $200 \mu \mathrm{m}$ ), $\mathbf{d}$ side view of Bio- $\mathrm{A}^{\circledR}$ at $\times 40$ magnification (scale bar $100 \mu \mathrm{m}$ )

and mesh thickness measurements are reported below as mean \pm standard deviation.

\section{Bacterial inoculation model (rabbit study)}

\section{Device implantation/inoculation/explantation}

An established rabbit bacterial inoculation model was utilized for the current study [7-9]. Approval was obtained from the Institutional Animal Care and Use Committee at WuXiAppTec, Inc. (St. Paul, MN). All animals were treated in accordance with the Guide for the Care and Use of Laboratory Animals. The study consisted of 20 male New Zealand White Rabbits (2.9-3.7 kg), divided into 2 groups, including fully resorbable synthetic [Bio-A ${ }^{\circledR}$ Tissue Reinforcement (Bio- $\left.\left.\mathrm{A}^{\circledR} ; n=10\right)\right]$ and fully resorbable biologically derived [Phasix ${ }^{\mathrm{TM}}$ Mesh (Phasix $\left.\left.{ }^{\mathrm{TM}} ; n=10\right)\right]$.

As described previously [7, 8], animals were initially anesthetized with 2.5-5\% inhalational isoflurane (to effect), and maintained at $0.5-5 \%$ (to effect) throughout the procedure. The dorsal area was prepared for aseptic survival surgery by shaving the entire midline region of the back, 

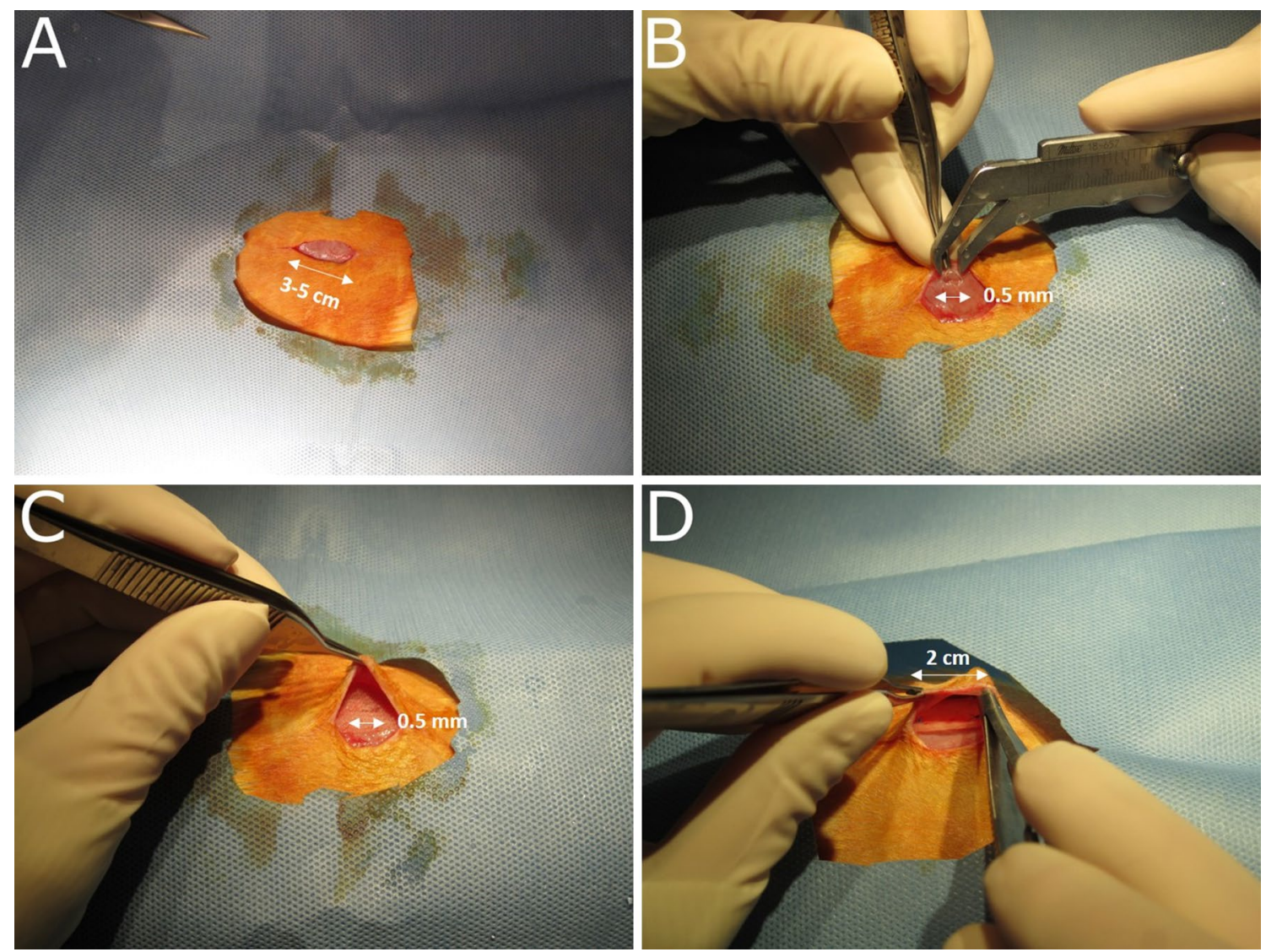

Fig. 2 Non-contaminated hernia defect model (rat study): device implantation a using sterile technique, a 3-5-cm midline incision was created on the ventral abdomen of each rat. The skin was dissected, revealing the subcutaneous plane. b, c A $0.5-\mathrm{cm}$ defect in the muscle

cleaning the operative area with three alternating scrubs of povidone-iodine/70\% isopropyl alcohol solutions, and then sterile surgical drapes were placed over the entire field. Following preparation of the dorsal surface for aseptic surgery, a $2.0-2.5-\mathrm{cm}$ lateral incision along the dorsal surface of the thoracic spine at the level of the scapulae was created through the dermal layer. A single incision was made parallel to the midline of the back, cutting through the fascia and exposing the paravertebral muscle. The fascial membrane was incised with a scalpel and enlarged using blunt dissection to create a pocket for implanted mesh. The implant pockets were created bilaterally along the lateral wall toward the lateral aspect of the scapula of each rabbit to accommodate insertion and placement of one test device (3.8-cm-diameter circle) per side into the subcutaneous space. One previously die-cut and soaked/rinsed (as appropriate according to the instructions for use for each device) implant was gently introduced into each of the subcutaneous pockets (Fig. 3a). Each animal received the same device in each of the bilateral pockets. The distal needle of a Vacutainer ${ }^{\circledR}$ blood collection set was then removed to layer was created using a scalpel. d A $2 \times 2 \mathrm{~cm}$ square of mesh was implanted into the subcutaneous pocket over the defect. Interrupted sutures were used to fixate the corners of the mesh to the abdominal wall, and the skin was then closed

create a bacterial injection catheter. The 1.5 -mm-diameter tubing was placed into each pocket between the device and the infraspinous fossa of the scapula. Through blunt dissection, a hemostatic clamp was used to create a tunnel from each implant pocket towards the caudal aspect, parallel to the midline, for approximately $7-10 \mathrm{~cm}$. The injection catheter tubing was retracted through this tunnel so that one end was in the implant pocket, and the other end was pulled through an incision made at the other end of the tunnel. This permitted remote inoculation of each pocket once the implantation procedure was complete. The tubing was temporarily secured with a purse-string suture. The pocket surrounding each device was sutured closed using a continuous pattern, reconnecting the subcutaneous tissues around the device. The cutaneous tissues were closed with a two-layer closure technique using absorbable sutures. Staples were also used to close the incision sites. Each device/isolated pocket was then inoculated with clinically isolated MRSA $(1.0 \mathrm{ml}$ of $\left.1 \times 10^{8} \mathrm{CFU} / \mathrm{ml}\right)$. Prepared syringes were used to deliver the determined dose into each pocket via the indwelling inoculation catheters, each followed by a $1.0 \mathrm{ml}$ flush of 


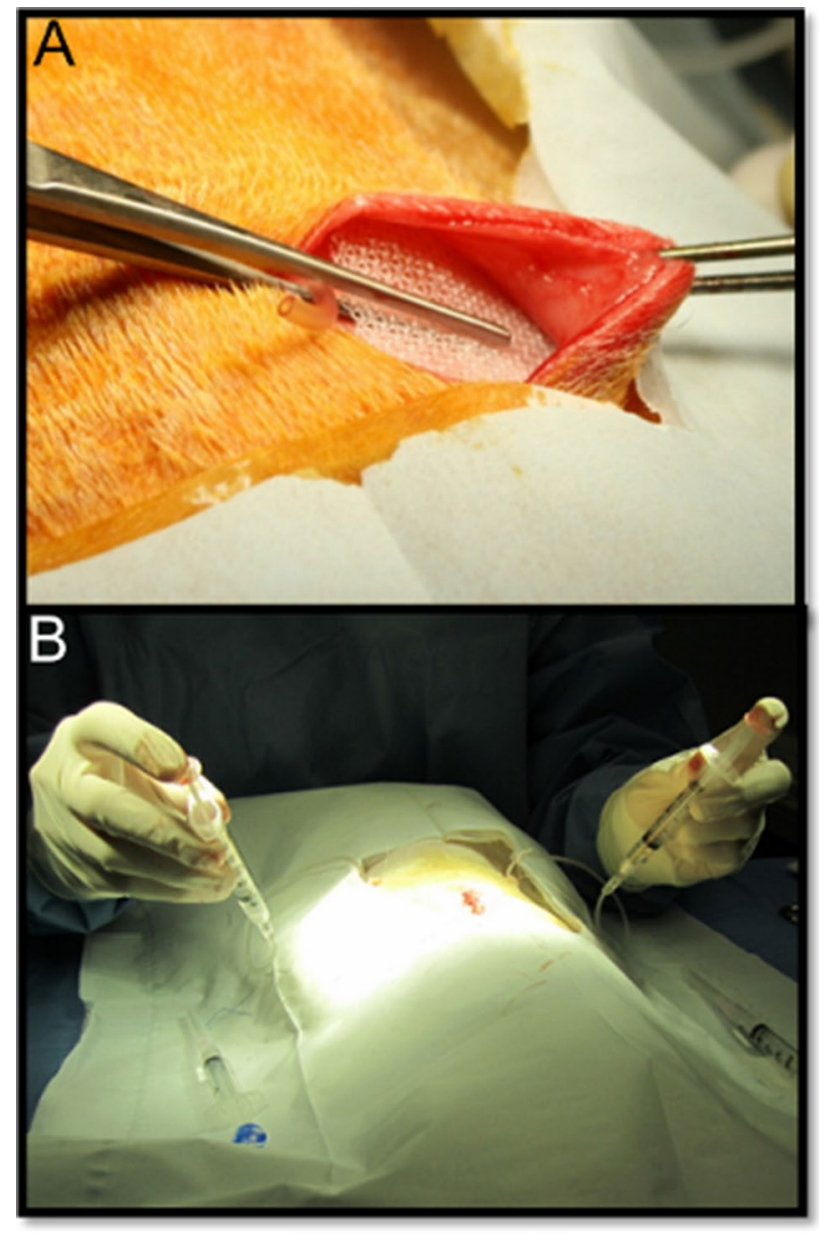

Fig. 3 Bacterial inoculation model (rabbit study): device implantation/inoculation. a Bilateral implant pockets were created along the lateral wall toward the lateral aspect of the scapula of each rabbit to accommodate insertion and placement of one device per side into the subcutaneous space and sutured closed. b Each device/isolated pocket was then inoculated with a controlled concentration of clinically isolated MRSA $\left(1.0 \mathrm{ml}\right.$ of $\left.1 \times 10^{8} \mathrm{CFU} / \mathrm{ml}\right)$

sterile saline from separate syringes (Fig. 3b). The catheters were subsequently removed and previously placed bilateral purse-string sutures were closed to seal each pocket. Animals were recovered from anesthesia and allowed free access to food and water. At 7 days post-implantation, euthanasia was achieved by intravenously injecting rabbits with $150 \mathrm{mg} / \mathrm{kg}$ of sodium pentobarbital, in accordance with the American Veterinary Medical Association (AVMA) Panel of Euthanasia. Following euthanasia, the overlying skin and adipose tissue were removed from the dorsal aspect of each animal aseptically, and implant locations were dissected to expose each device.
Abscess formation

Upon explantation, each device and device location was inspected for evidence of abscess formation. As described in previous studies $[7,8]$, abscess was independently scored by a trained investigator at the study site as: none (0), mild (1), moderate (2), or marked (3). The device from the left side of the animal was further analyzed for MRSA colonyforming units (CFU) within or adherent to the device, and the device from the right side of the animal was analyzed for mechanical (ball burst) strength.

\section{MRSA bacterial colonization analysis}

As described in previous studies [7-9], devices assigned to undergo bacterial colonization analysis were aseptically explanted, rinsed, vortexed for $30 \mathrm{~s}$ and sonicated for $5 \mathrm{~min}$ for a total of two sonication procedures. The sonicant solutions were serially diluted, $10^{-1}, 10^{-2}$, and $10^{-3}$, and the undiluted and diluted samples were plated on trypticase soy agar (TSA) plates to determine bacterial colonization of the device. All subcutaneous tissue pockets were also aseptically swabbed and streaked onto TSA plates to determine bacterial colonization of the implant pockets. For both device sonicant and subcutaneous tissue pocket swab specimens, TSA plates were incubated overnight at $37^{\circ} \mathrm{C}$ and examined for the presence of MRSA colonies. Pocket swab plates were characterized as positive if one or more MRSA colonies were identified and negative if no colonies were present. For device sonicant specimens, MRSA colonies were quantified, recorded, and the resulting CFU calculated as the sum of sonicant 1 and 2 .

\section{Mechanical analysis}

For devices assigned to undergo mechanical strength analysis, mechanical evaluations were accomplished using calibrated tensiometric equipment, including a servo-hydraulic testing system (Instron, Corp., Norwood, MA). All mechanical analyses were conducted by an independent biomaterial consulting firm (Altran Solutions, Boston, MA). An appropriate load cell $(2,000 \mathrm{~N})$ was selected such that the applied forces were within the operating range. To ensure adequate sample gripping characteristics, all loosely adhered tissue was removed so that the mechanical ball burst fixture could be tightly applied around the device prior to test initiation. From each device, Peak Ball Burst Force (N) (defined as the peak load recorded at test device failure) was captured by the computerized data acquisition system $(20 \mathrm{~Hz})$ as a 3/8-inch $(95 \mathrm{~mm})$-diameter ball interacted with the device in compression at a controlled displacement rate of $1 \mathrm{inch}$ $(25.4 \mathrm{~mm}) / \mathrm{min} . T_{0}$ (non-implanted) devices were identically 
evaluated to establish a pre-implantation baseline strength for each material.

\section{Statistical analysis}

For statistical comparisons, data were collected, analyzed, interpreted, and graphically displayed with GraphPad Prism ${ }^{\circledR}$ 6.01 statistical software (GraphPad Software Inc., La Jolla, CA). A nonparametric Kruskal-Wallis test with Dunn's post test was performed for inflammation scores. Data are presented as median with interquartile range $(25-75 \%)$. The threshold of statistical significance was set at $p<0.05$. A one-way ANOVA was performed for mesh thickness and repair thickness measurements, followed by Sidak's multiple comparison post test. Data are presented as mean \pm standard deviation. The threshold of statistical significance was set at $p<0.05$. A nonparametric Mann-Whitney test was performed for Abscess Score (0-3 point scale) and CFU (sum of sonicant 1 and 2 on a logarithmic scale). Data are presented as median with interquartile range $(25-75 \%)$. The threshold of statistical significance was set at $p<0.05$. Ball burst data were analyzed via one-way ANOVA, followed by Bonferroni post test. Data are presented as mean \pm standard deviation. The threshold of statistical significance was set at $p<0.05$. The number of MRSA-positive pocket swabs was analyzed via contingency table with Fisher's exact test. The threshold of statistical significance was set at $p<0.05$.

\section{Results}

\section{Non-contaminated hernia defect model (rat study)}

\section{Gross necropsy}

Photographs were taken of each implant site at each time point during necropsy, and representative images are shown in Fig. 4. The rapid resorption of Bio- $\mathrm{A}^{\circledR}$ is clearly evident at the later time points, particularly at 16 and 24 weeks, compared to the relatively unchanged appearance of the Phasix ${ }^{\mathrm{TM}}$ implants throughout the duration of the study.

\section{Inflammation}

As shown in Fig. 5, Phasix ${ }^{\mathrm{TM}}$ implant sites exhibited a median inflammation score of 2.0 at alltime points evaluated, representing a mild inflammatory response that remained consistent over time. Macrophages (mild) and lymphocytes (minimal to negligible) were consistent at all time points. Neutrophils and granulomas were minimal at 2 weeks and progressively decreased to negligible or absent at all subsequent time points. Eosinophils were minimal up to 8 weeks, progressively decreased thereafter, and were absent at

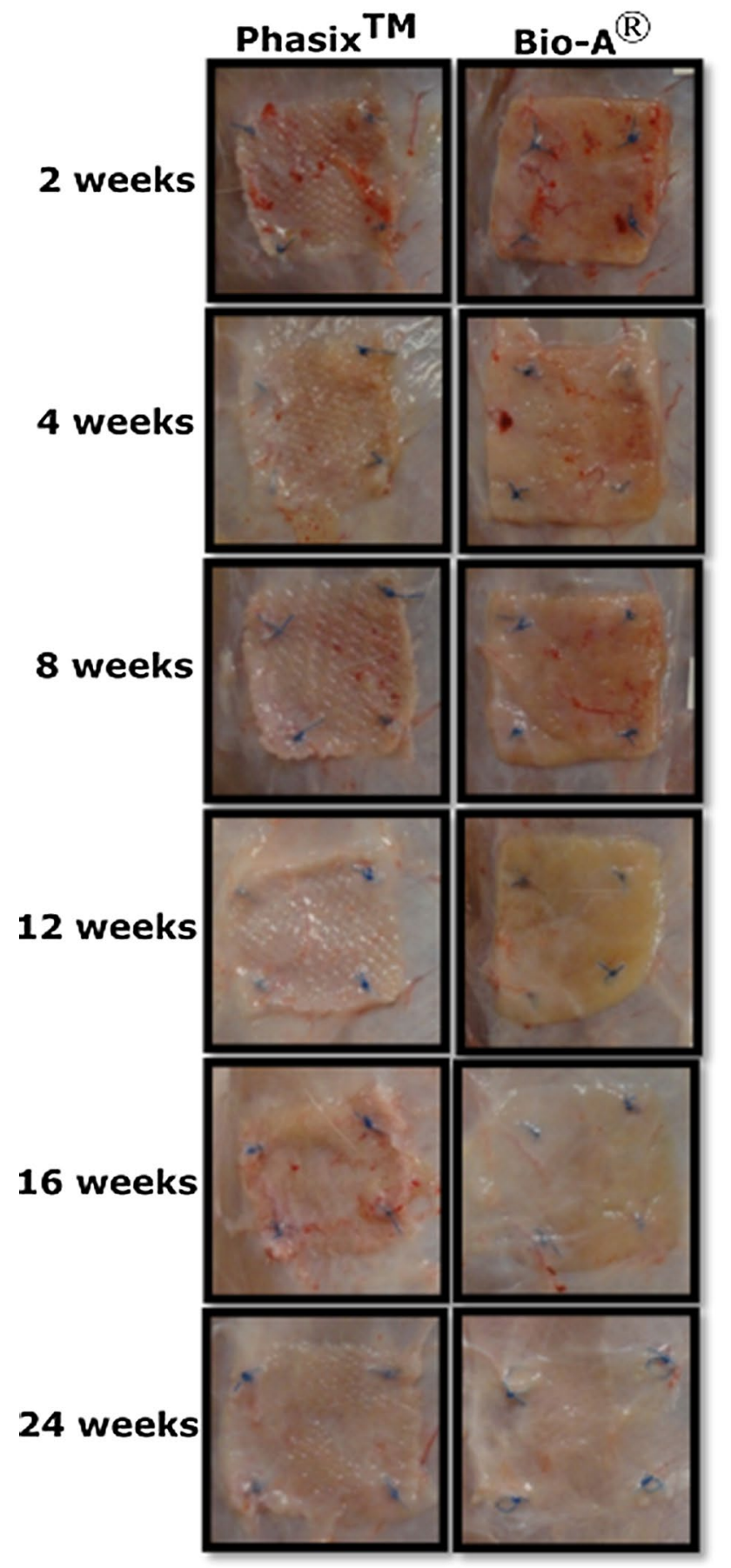

Fig. 4 Non-contaminated hernia defect model (rat study): representative gross necropsy photographs of Phasix ${ }^{\mathrm{TM}}$ sites (left column) and Bio-A ${ }^{\circledR}$ sites (right column) over time

24 weeks. Giant cells decreased from mild at 2 weeks to minimal-mild at all subsequent time points.

Bio- $\mathrm{A}^{\circledR}$ sites exhibited a median inflammation score of 4.0 at 2 and 4 weeks, indicating a marked inflammatory response. However, between 8 and 24 weeks, the median score for Bio- $\mathrm{A}^{\circledR}$ sites ranged from 2.0-3.0, indicating a 


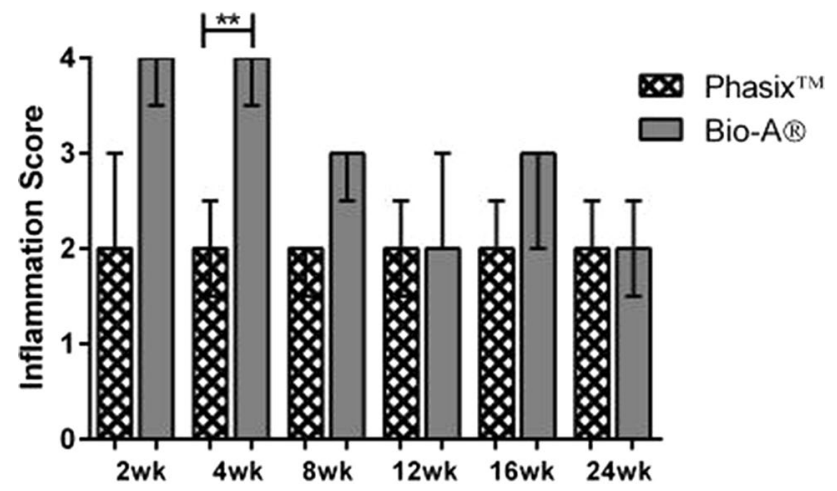

Fig. 5 Non-contaminated hernia defect model (rat study): inflammation scores for Phasix ${ }^{\mathrm{TM}}$ and Bio- $\mathrm{A}^{\circledR}$ sites over time. Bio- $\mathrm{A}^{\circledR}$ demonstrated significantly greater inflammation at 4 weeks compared to Phasix $^{\mathrm{TM}}(* * p<0.01)$

transition to a mild/moderate inflammatory response over time, upon bulk material resorption. Macrophages and eosinophils decreased progressively over time, with macrophages transitioning from moderate-marked to minimalmild and eosinophils transitioning from mild-moderate to absent. Neutrophils decreased from mild at 2 weeks to negligible or absent at all subsequent time points. Giant cells progressively decreased from moderate-marked at 2 weeks to minimal at 24 weeks. Lymphocytes (minimal-mild) remained consistent over time.

No significant differences in inflammatory response were observed between materials except at 4 weeks when Bio$\mathrm{A}^{\circledR}$ (median 4.0, interquartile range 3.5-4.0) demonstrated significantly greater inflammation compared to Phasix ${ }^{\mathrm{TM}}$ (median 2.0, interquartile range $1.5-2.5, p<0.01$ ).

\section{Repair thickness}

Mesh thickness (mm) and repair thickness (mesh thickness + surrounding fibrotic response, $\mathrm{mm}$ ) for Phasix ${ }^{\mathrm{TM}}$ and Bio- $\mathrm{A}^{\circledR}$ sites are depicted in Figs. 6 and 7 (mean \pm standard deviation). The pre-implantation thickness $\left(T_{0}\right)$ of Phasix ${ }^{\mathrm{TM}}$ has been previously reported as $0.51 \mathrm{~mm}(0.02 \mathrm{in})$ and is depicted as a dashed line in Fig. 7a [10]. As shown in the white bars of Fig. 7a, the thickness of Phasix ${ }^{\mathrm{TM}}$ mesh remained similar to its pre-implantation value and was relatively unchanged between time points throughout the study ( $p>0.05$ for all comparisons). However, Phasix ${ }^{\mathrm{TM}}$ mesh demonstrated an overall significant decrease in thickness at 24 weeks compared to 2 weeks $(p<0.05)$. As shown in the cross-hatched bars in Fig. 7b, the repair thickness (i.e., mesh thickness + surrounding fibrotic response) associated with Phasix ${ }^{\mathrm{TM}}$ sites was comparable between 2 and 4 weeks $(p>0.05)$ and then experienced minor, though statistically significant, changes between 4 and 24 weeks ( $p<0.0001$ for all comparisons between time points). However, the repair

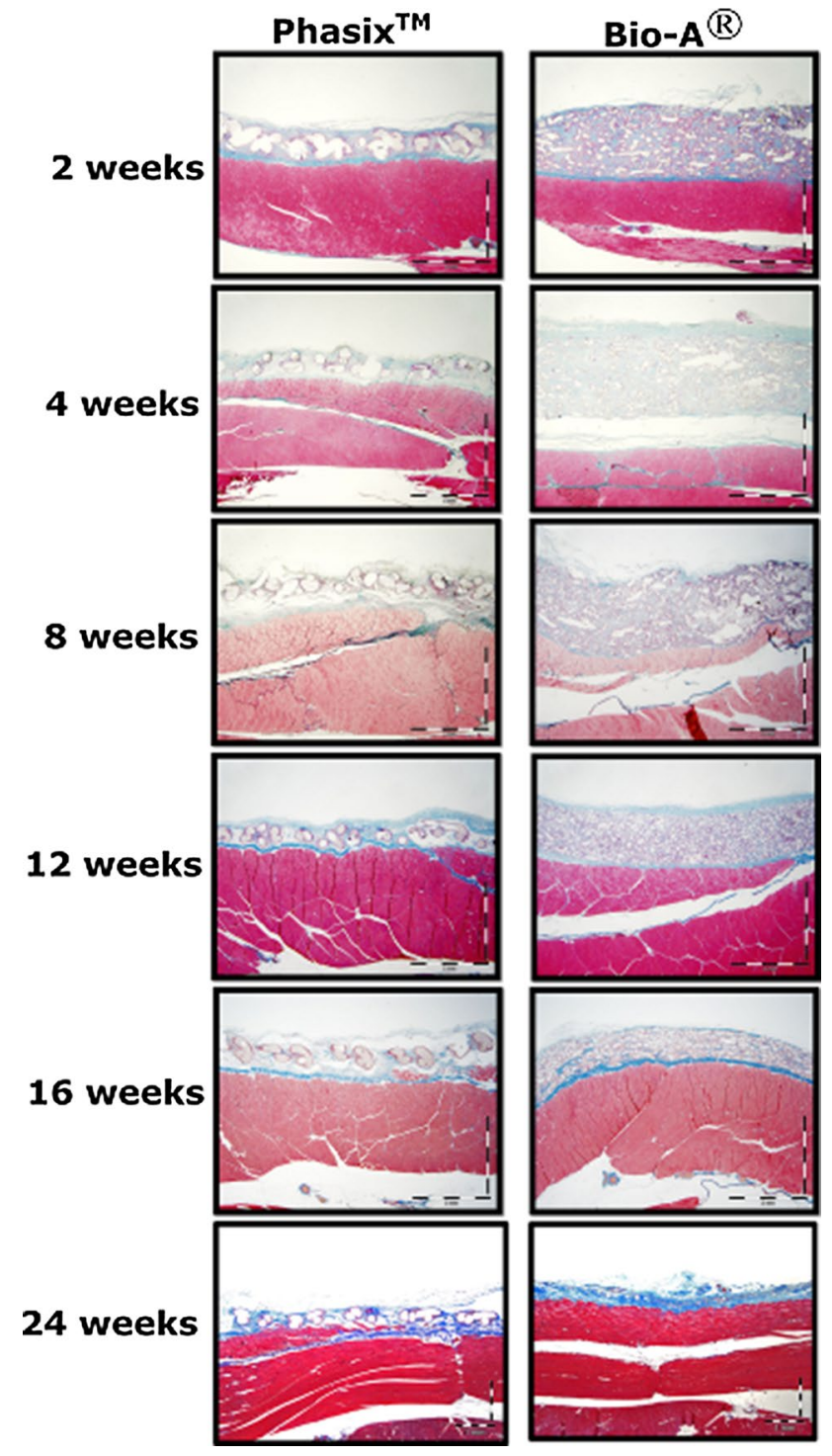

Fig. 6 Non-contaminated hernia defect model (rat study): representative histology photographs of Phasix ${ }^{\mathrm{TM}}$ sites (left column) and Bio$\mathrm{A}^{\circledR}$ sites (right column) over time (Masson's trichrome stain, $\times 1.25$ magnification)

thickness associated with Phasix ${ }^{\mathrm{TM}}$ sites at 24 weeks ultimately remained comparable to that measured at 2 weeks $(p>0.05)$.

The pre-implantation thickness of Bio- $\mathrm{A}^{\circledR}$ has been previously reported as $1.5 \mathrm{~mm}$ and is also depicted as a dashed line in Fig. 7a [11]. As shown in the dotted bars in Fig. 7a, the thickness of Bio- $\mathrm{A}^{\circledR}$ was similar to its pre-implantation value at 2,4 , and 8 weeks and then began to decline sharply thereafter. In fact, a significant increase in Bio- $\mathrm{A}^{\circledR}$ mesh thickness was observed between 2 and 4 weeks $(p<0.01)$, with no significant change between 4 and 8 weeks $(p>0.05)$, followed by significant decreases between all subsequent time points $(p<0.01$ for all comparisons between 8 and 


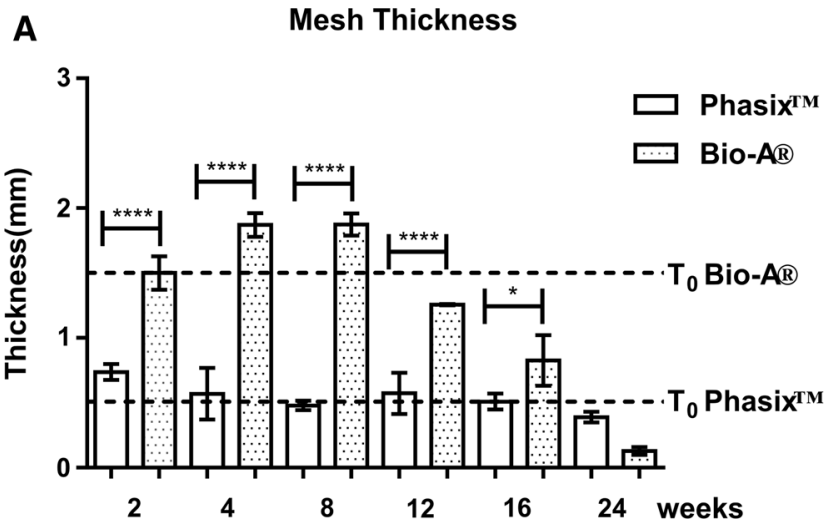

Fig. 7 Non-contaminated hernia defect model (rat study): a mesh thickness $(\mathrm{mm})$ and $\mathbf{b}$ repair thicknesses for Phasix ${ }^{\mathrm{TM}}$ and Bio- ${ }^{\circledR}$ sites (mesh thickness + surrounding fibrotic response, $\mathrm{mm}$ ) over

24 weeks). In addition, Bio- $A^{\circledR}$ mesh also demonstrated an overall significant decrease in mesh thickness at 24 weeks compared to 2 weeks $(p<0.0001)$. As shown in the gray bars of Fig. 7b, the repair thickness associated with Bio$\mathrm{A}^{\circledR}$ sites increased significantly between 2 and 4 weeks $(p<0.0001)$ and then decreased significantly between all subsequent time points $(p<0.0001$ for all comparisons between 4 and 24 weeks). Additionally, the repair thickness associated with Bio- $\mathrm{A}^{\circledR}$ sites showed an overall significant decrease at 24 weeks compared to 2 weeks $(p<0.0001)$.

When comparing mesh thicknesses reported for the two materials (Fig. 7a), Bio- $\mathrm{A}^{\circledR}$ mesh exhibited significantly greater thickness than Phasix ${ }^{\mathrm{TM}}$ mesh $(p<0.05$ for all comparisons) at all time points except 24 weeks when the thicknesses of the two materials were comparable $(p>0.05)$. In terms of the overall repair thickness (Fig. 7b), Bio- ${ }^{\circledR}$ sites demonstrated significantly greater repair thickness than Phasix $^{\mathrm{TM}}$ sites at $2,4,8$, and 12 weeks $(p<0.0001$ in all cases). However, at 16 and 24 weeks, Phasix ${ }^{\mathrm{TM}}$ sites demonstrated significantly greater repair thickness than Bio-A ${ }^{\circledR}$ sites $(p<0.0001$ and $p<0.01$, respectively).

\section{Bacterial inoculation model (rabbit study)}

\section{Gross necropsy}

It should be noted that inadvertent contamination of one specimen occurred that affected the Phasix ${ }^{\mathrm{TM}}$ implant on the left side of one animal. Bacterial colonization analysis, pocket swabs, and abscess scores were not recorded for this specimen and, as such, were not included in the final data analysis. Staphylococcus lentus (S. lentus) was recovered from this implant. No other implant sites had S. lentus cultured from samples collected. Confirmation plating from inoculums pre- and post-implant indicates
B

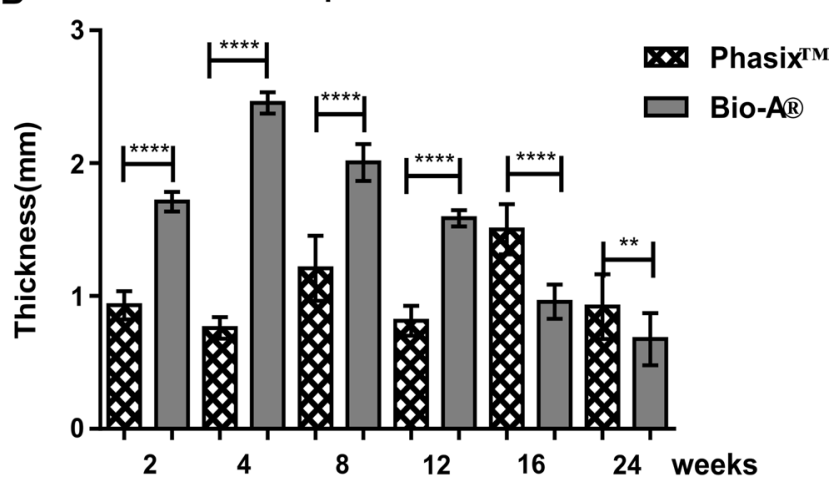

time compared to pre-implantation $\left(T_{0}\right)$ mesh thickness, $* p<0.05$, $* * p<0.01, * * * * p<0.0001$

that the contamination did not originate from the bacteria inoculum.

\section{Abscess formation}

Fibrinous exudate (FE) and pus is primarily comprised of residual bacterial and leukocyte cellular debris and is indicative of a recent or ongoing inflammatory response. As shown in Fig. 8a, Bio- $\mathrm{A}^{\circledR}$ demonstrated extensive FE formation on the device and within the implant pocket compared to Phasix ${ }^{\mathrm{TM}}$. As shown in Fig. 8b, at 7 days postimplantation/inoculation, Bio- ${ }^{\circledR}$ also demonstrated a significantly higher abscess score (median 3.0, interquartile range 1.63-3.0) representing marked abscess compared to mild abscess observed for Phasix ${ }^{\mathrm{TM}}$ (median 1.0, interquartile range $0.0-1.0, p<0.001)$.

\section{MRSA bacterial colonization analysis}

As shown in Table 1, Phasix ${ }^{\mathrm{TM}}$ demonstrated significantly lower MRSA bacterial colonization (median 0.00 CFU, interquartile range $0.00-3.75 \times 10^{2} \mathrm{CFU}$ ) compared to Bio- $\mathrm{A}^{\circledR}$ (median $2.78 \times 10^{7} \mathrm{CFU}$, interquartile range $\left.1.31 \times 10^{3}-5.58 \times 10^{7} \mathrm{CFU}, p<0.01\right)$ at 7 days postimplantation/inoculation. Also shown in Table 1, Phasix ${ }^{\mathrm{TM}}$ demonstrated significantly fewer MRSA-positive pocket swabs $(4 / 18,22 \%)$ compared to Bio- $A^{\circledR}(16 / 20,80 \%$, $p<0.001)$ at 7 days post-implantation/inoculation.

\section{Mechanical analysis}

As shown in Fig. 9a, Phasix ${ }^{\mathrm{TM}}$ at $T_{0}$ (non-implanted) exhibited significantly greater ball burst strength $(186.3 \pm 17.1 \mathrm{~N}$, mean \pm standard deviation) than Bio- ${ }^{\circledR}(120.6 \pm 16.9 \mathrm{~N}$, 

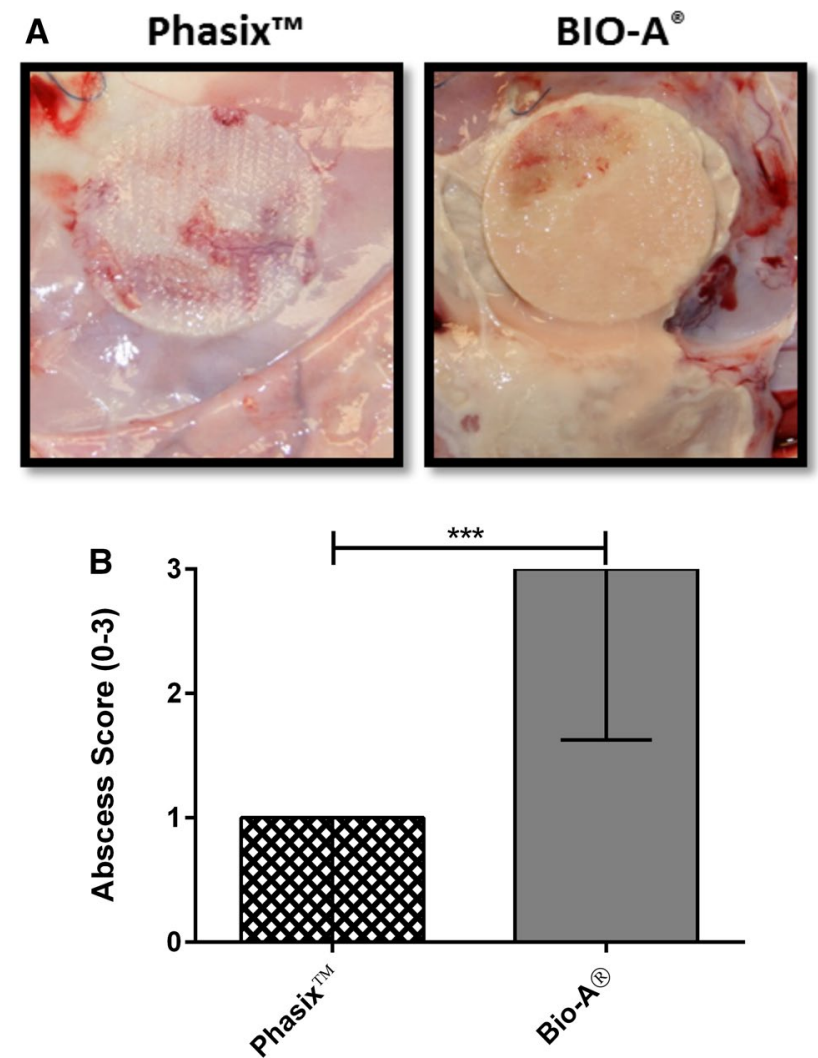

Fig. 8 Bacterial inoculation model (rabbit study): a representative gross necropsy photographs at 7 days post-implantation/inoculation with MRSA $\left(1 \times 10^{8} \mathrm{CFU} / \mathrm{ml}\right)$. Bio- ${ }^{\circledR}$ demonstrated elevated amounts of white material (abscess) present on the device and within the implant pocket compared to Phasix ${ }^{\mathrm{TM}}$. b White material (abscess) scoring. Bio- ${ }^{\circledR}$ demonstrated significantly higher white material (abscess) scores compared to Phasix ${ }^{\mathrm{TM}} 7$ days post-implantation/inoculation with MRSA $(* * * p<0.001)$

Table 1 Bacterial inoculation model (rabbit study)

\begin{tabular}{lll}
\hline & CFU median (IQR) & Positive swabs \\
\hline Phasix $^{\text {TM }}$ & $0.00 * *\left(0.00-3.75 \times 10^{2}\right)$ & $4 / 18^{* * *}(22 \%)$ \\
Bio-A $^{\circledR}$ & $2.78 \times 10^{7}\left(1.31 \times 10^{3}-5.58 \times 10^{7}\right)$ & $16 / 20(80 \%)$ \\
\hline
\end{tabular}

Total MRSA CFU on explanted devices and MRSA-positive pocket swabs. Phasix ${ }^{\mathrm{TM}}$ demonstrated significantly lower MRSA bacterial colonization (CFU) compared to Bio-A ${ }^{\circledR}(* * p<0.01)$ Phasix ${ }^{\mathrm{TM}}$ demonstrated significantly fewer MRSA-positive pocket swabs compared to Bio-A ${ }^{\circledR}(* * * p<0.001)$

$p<0.001)$. At 7 days post-implantation/MRSA inoculation, Phasix ${ }^{\mathrm{TM}}$ exhibited a significantly greater ball burst strength $(162.3 \pm 32.1 \mathrm{~N})$ than Bio- $\mathrm{A}^{\circledR}(10.6 \pm 5.9 \mathrm{~N}, p<0.001)$, as shown in Fig. 9b. Compared to $T_{0}$ (non-implanted) strength values, Phasix ${ }^{\mathrm{TM}}$ and Bio- $\mathrm{A}^{\circledR}$ demonstrated a 13 and $91 \%$ strength reduction, respectively $(p<0.05 ; p<0.001)$ at 7 days post-implantation, as shown in Fig. $9 \mathrm{c}$.

\section{Discussion}

The fully resorbable (synthetic and biologically sourced) scaffolds evaluated in this study currently represent commercially available options for abdominal wall reconstruction in which permanent foreign material retention may not be desired. Phasix ${ }^{\mathrm{TM}}$ mesh is comprised of poly-4-hydroxybutyrate $(\mathrm{P} 4 \mathrm{HB})$ monofilament that has been knitted to create a fully resorbable macroporous mesh. P4HB is not chemically synthesized, but rather is produced inside E. coli $\mathrm{K} 12$ bacteria through a transgenic fermentation process and then extracted. P4HB degrades via hydrolysis, and the resulting byproducts are eliminated from the body through the Krebs cycle [10]. Bio- ${ }^{\circledR}$ is comprised of a copolymer of poly (glycolide:trimethylene carbonate) fibers that form a three-dimensional webbed structure. The fibers are chemically synthesized rather than biologically derived and degrade through hydrolytic and enzymatic mechanisms after approximately 6 months in vivo [12].

This study was designed to provide fundamental insight into the impact that fully resorbable biomaterial/morphological features have on the resulting host tissue response, resorption profile, repair thickness, and mechanical strength properties, and to assess the performance in the presence of bacteria for both macroporous/monofilament (Phasix ${ }^{\mathrm{TM}}$ ) and microporous/multifilament/sheet-like (Bio$\mathrm{A}^{\circledR}$ ) designs. To accomplish this assessment, both non-contaminated and contaminated models were employed. The non-contaminated hernia defect model (rats) was primarily utilized to evaluate both fully resorbable biomaterials from a macroscopic and microscopic perspective over an extended period of time (up to 24 weeks), whereas the bacterial inoculation model (rabbits) was specifically utilized to evaluate properties of each material associated with bacterial colonization following direct inoculation with clinically isolated MRSA, and to determine the resulting impact on strength.

\section{Non-contaminated hernia defect model (rat study)}

Phasix ${ }^{\mathrm{TM}}$ and Bio- $\mathrm{A}^{\circledR}$ exhibited similar biocompatibility overall, although distinct temporal differences were observed. Specifically, Bio- $\mathrm{A}^{\circledR}$ demonstrated a heightened host inflammatory response at early time points (2-4 weeks), which was significantly greater than Phasix ${ }^{\mathrm{TM}}$ at 4 weeks post-implantation $(p<0.01)$. Possessing a microporous/ multifilament/sheet-like structure, Bio- $\mathrm{A}^{\circledR}$ scaffolds contain a vastly greater surface area compared to the macroporous/monofilament structure of Phasix ${ }^{\mathrm{TM}}$. From a cellular perspective, greater macrophage infiltration was observed within the Bio- $\mathrm{A}^{\circledR}$ scaffold architecture which surrounded the numerous compressed multifilament fibers at 2 and 
Fig. 9 Bacterial inoculation model (rabbit study): mechanical (ball burst) strength. a At $T_{0}$, Phasix ${ }^{\mathrm{TM}}$ exhibited significantly greater ball burst strength than Bio-A $^{\circledR}(p<0.001)$. b After exposure to MRSA, $T_{7 \text { day }}$ Phasix $^{\mathrm{TM}}$ exhibited significantly greater ball burst strength than $T_{7 \text { day }}$ Bio- $^{\circledR}(p<0.001)$. c Between $T_{0}$ and 7 days, a significant reduction in mechanical strength was demonstrated for both Phasix ${ }^{\mathrm{TM}}(-13 \%, p<0.05)$ and Bio- $\mathrm{A}^{\circledR}(-91 \%, p<0.001)$

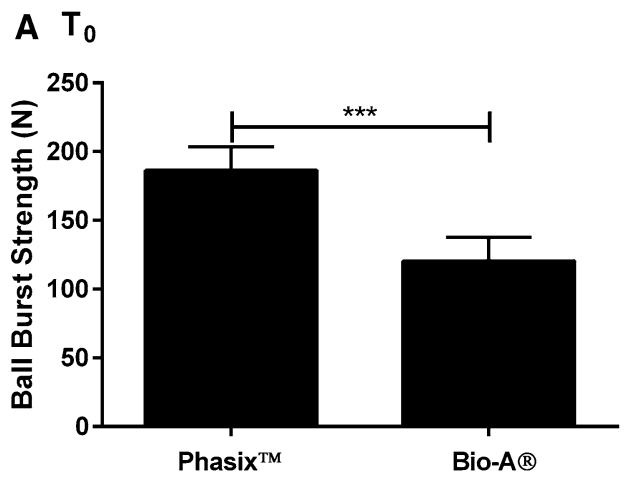

B 7 day

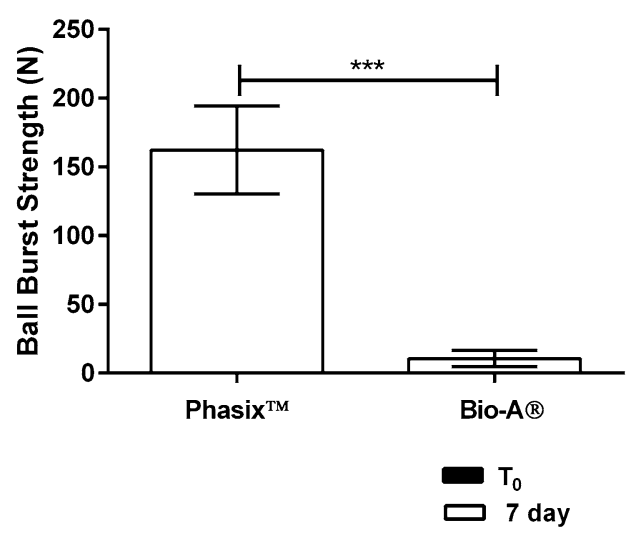

4 weeks, and may explain the increased inflammatory response at the earlier time points.

Both gross necropsy and histological assessment indicated a more rapid resorption profile for Bio- $\mathrm{A}^{\circledR}$ compared to Phasix ${ }^{\mathrm{TM}}$. Bio- $\mathrm{A}^{\circledR}$ exhibited unique temporal resorption dynamics, including fissuring of the scaffold by 8 weeks, substantial thinning by 12 weeks, and bulk resorption between 16 and 24 weeks. Bio-A ${ }^{\circledR}$ resorption was deemed essentially complete by 24 weeks post-implantation, which is fitting with its profile. Phasix ${ }^{\mathrm{TM}}$ has previously been demonstrated to be essentially completely resorbed by 72 weeks post-implantation $[10,13]$. The medium-term duration of this evaluation is an acknowledged limitation of this study, although the primary goal was to compare/contrast differences between both biomaterials at identical time points.

Histological/morphometric analysis also demonstrated unique differences in the overall repair thicknesses associated with Bio- $\mathrm{A}^{\circledR}$ and Phasix ${ }^{\mathrm{TM}}$ sites over time. For example, Bio- $\mathrm{A}^{\circledR}$ initially exhibited thicker repair sites at $2,4,8$, and 12 weeks $(p<0.0001)$ that transitioned to significantly thinner sites at 16 and 24 weeks compared to Phasix $^{\mathrm{TM}}(p<0.0001)$. Additional studies are required to assess the clinical significance of these results and to determine whether a thicker repair represents a more favorable outcome.

It should be noted that the overall repair thickness measurement included the thickness of the mesh itself, as well as that of the surrounding fibrotic host tissue response. Throughout the time points there was a $61 \%$ reduction in repair site thickness between 2 and 24 weeks for Bio$\mathrm{A}^{\circledR}$ compared to a $1 \%$ reduction for Phasix ${ }^{\mathrm{TM}}$. Because the thickness of the Phasix ${ }^{\mathrm{TM}}$ mesh itself remained relatively unchanged over time in this study, it is likely that increases in host tissue thickness were responsible for fluctuations in overall repair thickness observed for Phasix ${ }^{\mathrm{TM}}$ sites throughout the course of the study. At 24 weeks, the Phasix ${ }^{\mathrm{TM}}$ mesh thickness decreased significantly relative to 2 weeks. Thus, the host tissue contributed even more at 24 weeks, which kept the overall repair thickness comparable to 2 weeks. The mesh thickness and overall repair thickness measurements for Bio- $\mathrm{A}^{\circledR}$ sites exhibited similar trends throughout the course of the study, with an initial increase in thickness at the early time points, followed by a sustained decrease in thickness throughout the remainder of the study. Thus, it is likely that the trend for overall repair thickness observed for Bio- $\mathrm{A}^{\circledR}$ sites was driven primarily by changes in mesh thickness, while the contribution from the host tissue remained relatively constant over time compared to an increased host tissue contributing to the repair thickness over time for Phasix ${ }^{\mathrm{TM}}$. It is likely that cellular infiltration and swelling of the Bio- $\mathrm{A}^{\circledR}$ device led to the observed initial increase in mesh thickness which was reduced over time as the Bio- $\mathrm{A}^{\circledR}$ devices degraded. A limitation of this study is that the repair thickness measurements provide only partial information about the collagen deposited at the repair site. Gruber-Blum et al. have recently published a method that describes how the pattern of collagen deposition is related to the quality of the newly formed tissue, which may be 
used to better characterize the host response in future studies [14].

\section{Bacterial inoculation model (rabbit study)}

To evaluate the potential impact of microbial colonization on the fully resorbable scaffolds (if inadvertent contamination were to occur) an established rabbit bacterial inoculation model was utilized. A clinically isolated strain of methicillin-resistant Staphylococcus aureus (MRSA) was selected to most appropriately model the impact of a clinically significant device infection. Staphylococcus aureus (SA) represents the most common cause of surgical site infection and can also become a major cause of infection within the bloodstream [15]. Manunga et al. describe that of 1039 elective surgical patients, 48 (4.6\%) tested positive for MRSA (by nasal or oral swab) prior to surgical procedures, of which 3 (6.25\%) developed postoperative surgical site infections despite aggressive antibiotic treatment [16]. MRSA represents a particularly virulent drug-resistant form of Staphylococcus aureus, able to secrete a complex extracellular matrix biofilm, resulting in an extremely difficult pathogen to eradicate from prosthetic devices [17]. In the case of hernia repair, prosthetic device infection continues to represent a serious complication, correlated with greater patient morbidity, hernia recurrence, and incidence of reoperation [18, 19].

Abscess formation is indicative of a recent or ongoing host inflammatory response to the initial bacterial stimulus, resulting in accumulated cellular debris, which can inhibit integration of the device into the abdominal wall [20]. Data from the rabbit bacterial inoculation model indicated a strong impact of morphological features of the meshes studied on bacterial colonization and the resulting host response. Animals implanted with the microporous/multifilament/sheet-like Bio-A ${ }^{\circledR}$ scaffold exhibited significantly greater $(p<0.001)$ abscess and fibrinous exudate formation compared to the macroporous/monofilament Phasix ${ }^{\mathrm{TM}}$. Phasix $^{\text {TM }}$ demonstrated significantly lower viable MRSA $\mathrm{CFU}$ on the device (median $0.00 \mathrm{CFU}$; interquartile range $0.00-3.75 \times 10^{2} \mathrm{CFU}$ ) and a lower percentage of MRSApositive pocket swabs within the tissues surrounding the device $(22 \%)$ compared to Bio- $\mathrm{A}^{\circledR}$ (median $2.78 \times 10^{7} \mathrm{CFU}$; interquartile range $1.31 \times 10^{3}-5.58 \times 10^{7} \mathrm{CFU} ; p<0.01$ and $80 \%$ positive swabs; $p<0.001)$. Engelsman et al. have previously demonstrated that morphological elements such as multifilament versus monofilament designs impact the incidence of device infection [2]. Similarly, Harrell et al. demonstrated that lighter weight monofilament polypropylene meshes were less colonized following direct inoculation with MRSA compared to those comprised of multifilament permanent and/or multifilament absorbable design components
[3]. Sadava et al. also demonstrated that uncoated, monofilament polypropylene mesh was better able to resist MRSA bacterial adherence and biofilm formation than multifilament polyester mesh [17]. The primary reason for this has been described by Klinge et al. who demonstrated that the increased surface area associated with multifilament biomaterials enables significantly greater bacterial adherence compared to monofilament biomaterials [4]. Taken together, these data suggest that device design and overall surface area may greatly influence bacterial colonization regardless of material type, and should both be taken into consideration when selecting an appropriate prosthetic for patients with a higher risk of bacterial seeding of mesh and/or colonization.

Both fully resorbable scaffolds evaluated in this study exceeded the baseline strength requirements of a mesh material deemed appropriate for hernia repair applications [12, 21]. However, the rabbit model within the current study was utilized to determine the impact of in vivo implantation with direct bacterial inoculation on the mechanical properties of Phasix $^{\mathrm{TM}}$ and Bio-A ${ }^{\circledR}$. At 7 days, a 13 and $91 \%$ reduction in mechanical strength was observed for Phasix ${ }^{\mathrm{TM}}$ and Bio-A ${ }^{\circledR}$ scaffolds, respectively. Non-inoculated specimens were not included in the study design, which is another acknowledged limitation of the current study.

The results of the current study are in agreement with those presented in previous preclinical studies. Deeken et al. reported that Phasix ${ }^{\mathrm{TM}}$ maintained mechanical properties and continued to augment the strength of the porcine abdominal wall up to 52 weeks post-implantation in a noncontaminated porcine model [12]. These observations suggest that Phasix ${ }^{\mathrm{TM}}$ provides a robust repair that supports the defect site through the early healing phase and augments the strength of the abdominal wall even as the polymer is undergoing active resorption. The results of the current study also suggest that the mechanical properties of Phasix ${ }^{\mathrm{TM}}$ are minimally impacted by bacteria, but instead by predictable hydrolytic degradation over time. This is supported by another previous preclinical study. Martin et al. reported a stepwise (primarily hydrolytic) reduction in the mechanical strength of Phasix ${ }^{\mathrm{TM}}$ over a 72-week study period in a noncontaminated porcine model, which strongly correlated with a reduction in poly-4-hydroxybutyrate polymer molecular weight (MW) [10].

\section{Conclusions}

In summary, host tissue response, resorption profile, repair thickness, strength, and bacterial colonization data suggest a more favorable outcome for fully resorbable monofilament, macroporous devices such as Phasix ${ }^{\mathrm{TM}}$ relative to multifilament, sheet-like devices such as Bio- $\mathrm{A}^{\circledR}$. 


\section{Compliance with ethical standards}

Conflict of interest This study was funded by C. R. Bard, Inc. (Davol), Warwick, RI. Nathaniel F. N. Stoikes, MD, Corey R. Deeken, $\mathrm{PhD}$, and Guy R. Voeller, MD, FACS are consultants for, and Jeffrey R. Scott, PhD and Amit Badhwar, PhD are employees of C. R. Bard, Inc. (Davol), Warwick, RI. A portion of this study was also presented at the 15th Annual Hernia Repair conference of the Americas Hernia Society, Orlando, FL, March 2013.

Ethical approval All applicable international, national, and/or institutional guidelines for the care of animals were followed. All procedures performed in the studies involving animals were in accordance with the ethical standards of the institution or practice at which the studies were conducted.

Human and animal rights All animals were treated in accordance with the Guide for the Care and Use of Laboratory Animals. This article does not contain any studies with human participants performed by any of the authors.

Informed consent For this type of study informed consent was not required.

Open Access This article is distributed under the terms of the Creative Commons Attribution-NonCommercial 4.0 International License (http://creativecommons.org/licenses/by-nc/4.0/), which permits any noncommercial use, distribution, and reproduction in any medium, provided you give appropriate credit to the original author(s) and the source, provide a link to the Creative Commons license, and indicate if changes were made.

\section{References}

1. Cole WC, Balent EM, Masella PC, Kajiura LN, Matsumoto KW, Pierce LM (2014) An experimental comparison of the effects of bacterial colonization on biologic and synthetic meshes. Hernia 19:197-205

2. Engelsman AF, Van Dam GM, Van Der Mei HC, Busscher HJ, Ploeg RJ (2010) In vivo evaluation of bacterial infection involving morphologically different surgical meshes. Ann Surg 251:133-137

3. Harrell AG, Novitsky YW, Kercher KW, Foster M, Burns JM, Kuwada TS, Heniford BT (2006) In vitro infect ability of prosthetic mesh by methicillin-resistant Staphylococcus aureus. Hernia 10:120-124

4. Klinge U, Junge K, Spellerberg B, Piroth C, Klosterhalfen B, Schumpelick V (2002) Do multifilament alloplastic meshes increase the infection rate? Analysis of the polymeric surface, the bacteria adherence, and the in vivo consequences in a rat model. J Biomed Mater Res 63:765-771

5. Harth KC, Broome AM, Jacobs MR, Blatnik JA, Zeinali F, Bajaksouzian S, Rosen MJ (2011) Bacterial clearance of biologic grafts used in hernia repair: an experimental study. Surg Endosc 25:2224-2229
6. Deeken CR, Melman L, Jenkins ED, Greco SC, Frisella MM, Matthews BD (2011) Histologic and biomechanical evaluation of crosslinked and non-crosslinked biologic meshes in a porcine model of ventral incisional hernia repair. J Am Coll Surg 212:880-888

7. Majumder A, Scott J, Novitsky Y (2016) Evaluation of the antimicrobial efficacy of a novel Rifampin/Minocycline-coated, noncrosslinked porcine acellular dermal matrix compared to uncoated scaffolds for soft tissue repair. Surg Innov 23:442-455

8. Cohen L, Imahiyerobo T, Scott J, Spector J (2016) Comparison of antibiotic-coated versus uncoated porcine dermal matrix. Plast Reconstr Surg 138:844e-855e

9. Hansen LK, Berg K, Johnson D, Sanders M, Citron M (2010) Efficacy of local rifampin/minocycline delivery (AIGIS(RX)(R)) to eliminate biofilm formation on implanted pacing devices in a rabbit model. Int J Artif Organs 33:627-635

10. Martin DP, Badhwar A, Shah DV, Rizk S, Eldridge SN, Gagne DH, Ganatra A, Darois RE, Williams SF, Tai HC, Scott JR (2013) Characterization of poly-4-hydroxybutyrate mesh for hernia repair applications. J Surg Res 184:766-773

11. Yeo KK, Park TH, Park JH, Chang CH, Kim JK, Seo SW (2014) Histologic changes of implanted gore bio-A in an experimental animal model. Biomed Res Int 2014:167962

12. Deeken CR, Matthews BD (2013) Characterization of the mechanical strength, resorption properties, and histologic characteristics of a fully absorbable material (poly-4-hydroxybutyratePHASIX Mesh) in a porcine model of hernia repair. ISRN Surg 2013:238067

13. Phasix Mesh Instructions for Use, C. R. Bard (Davol), accessed electronically

14. Gruber-Blum S, Brand J, Keibl C, Fortelny RH, Redl H, Mayer F, Petter-Puchner AH (2017) Abdominal wall reinforcement: biologic vs. degradable synthetic devices. Hernia 21:305-315

15. Humphreys H (2012) Staphylococcus aureus: the enduring pathogen in surgery. Surgeon 10:357-360

16. Manunga J Jr, Olak J, Rivera C, Martin M (2012) Prevalence of methicillin-resistant Staphylococcus aureus in elective surgical patients at a public teaching hospital: an analysis of 1039 patients. Am Surg 78:1096-1099

17. Sadava EE, Krpata DM, Gao Y, Novitsky YW, Rosen MJ (2013) Does presoaking synthetic mesh in antibiotic solution reduce mesh infections? An experimental study. J Gastrointest Surg 17:562-568

18. Leber GE, Garb JL, Alexander AI, Reed WP (1998) Long-term complications associated with prosthetic repair of incisional hernias. Arch Surg 133:378-382

19. Engelsman AF, Van Der Mei HC, Ploeg RJ, Busscher HJ (2007) The phenomenon of infection with abdominal wall reconstruction. Biomaterials 28:2314-2327

20. Law NW, Ellis H (1991) A comparison of polypropylene mesh and expanded polytetrafluoroethylene patch for the repair of contaminated abdominal wall defects-an experimental study. Surgery 109:652-655

21. Ebersole GC, Buettmann EG, Macewan MR, Tang ME, Frisella MM, Matthews BD, Deeken CR (2012) Development of novel electrospun absorbable polycaprolactone (PCL) scaffolds for hernia repair applications. Surg Endosc 26:2717-2728 